\title{
Serum Neutralizing Antibody Response to the Vacuolating Cytotoxin of Helicobacter pylori
}

\author{
Timothy L. Cover, *‡ Ping Cao, ${ }^{*}$ Uma K. Murthy," Michael S. Sipple," and Martin J. Blaser ${ }^{\star \star s}$ \\ ${ }^{*}$ Infectious Disease Section, Department of Veterans Affairs Medical Center, and Departments of ${ }^{\ddagger}$ Medicine and ${ }^{\S}$ Microbiology and \\ Immunology, Vanderbilt University School of Medicine, Nashville, Tennessee 37232; and "Gastroenterology Section, Department of \\ Veterans Affairs Medical Center, and Department of Gastroenterology, University Hospital, Syracuse, New York 13210
}

\begin{abstract}
Approximately $\mathbf{5 0 \%}$ of Helicobacter pylori isolates produce a cytotoxin in vitro that induces vacuolation of eukaryotic cells. To determine the in vivo relevance of this phenomenon, we sought to detect cytotoxin-neutralizing antibodies in sera from $\mathrm{H}$. pylori-infected persons. As a group, sera from $29 \mathrm{H}$. pyloriinfected patients neutralized the activity of the purified cytotoxin to a significantly greater extent than sera from 24 uninfected persons $(P=0.007)$. The cytotoxin neutralizing activity in sera from $H$. pylori-infected persons was mediated predominantly by the purified IgG fraction. Sera from $H$. pylori-infected persons neutralized the cytotoxins produced by multiple $H$. pylori strains, but failed to neutralize trimethylamine-induced cell vacuolation. Neutralization of cytotoxin activity by human or immune rabbit sera was associated with immunoblot IgG recognition of an 87-kD $\mathrm{H}$. pylori protein. Similarly, neutralization of the toxin by sera was associated with IgG recognition of the purified cytotoxin in an enzyme-linked immunosorbent assay $(P<0.0001)$. The presence of cytotoxin-neutralizing antibodies in sera from $H$. pylori-infected persons indicates that the cytotoxin is synthesized in vivo. ( J. Clin. Invest. 1992. 90:913-918.) Key words: autophagy • gastritis • peptic ulcer • toxin • vacuole
\end{abstract}

\section{Introduction}

Helicobacter pylori infection is now recognized as the predominant cause of chronic gastritis in humans, and is strongly associated with peptic ulcer disease $(1,2)$. The pathogenic mechanisms whereby $H$. pylori causes human disease remain poorly understood. One potential determinant of virulence is a vacuolating cytotoxin that is detectable in broth culture supernatants from $\sim 50 \%$ of $H$. pylori strains (3-5). In vitro, this toxin induces vacuolation of multiple eukaryotic cell types (4). The vacuolating cytotoxin of $H$. pylori is distinct from urease, but is

This study was presented in part at the 92nd Annual Meeting of the American Gastroenterological Association, New Orleans, LA, 19-22 May 1991 (Abstract 445), and the 31st Interscience Conference on Antimicrobial Agents and Chemotherapy, Chicago, IL, 29 September2 October 1991 (Abstract 427).

Dr. Sipple's present address is Suite 809, 725 Irving Avenue, Syracuse, NY 13210.

Address reprint requests to Dr. Cover, Division of Infectious Diseases, A-3310 Medical Center North, Vanderbilt University School of Medicine, Nashville, TN 37232-2605.

Received for publication 3 October 1991 and in revised form 25 February 1992.

The Journal of Clinical Investigation, Inc.

Volume 90, September 1992, 913-918 potentiated by urease-mediated ammonia production (6). Recently, the cytotoxin has been purified, and migrates as an 87 $\mathrm{kD}$ band under denaturing conditions (7).

The role of the $H$. pylori-vacuolating cytotoxin in vivo has not yet been studied in detail. In gnotobiotic piglets experimentally infected with $H$. pylori, challenge with a toxigenic strain results in significantly more gastric epithelial vacuolation than challenge with a nontoxigenic strain (8), which suggests that the cytotoxin is active in vivo. Several histopathologic studies have identified vacuoles in the gastric epithelial cells of humans infected with $H$. pylori (9-11). However, it is not known whether these vacuoles form in response to $H$. pylori cytotoxin, ammonia, or other factors. Patients infected with a variety of noninvasive toxigenic bacteria, including Vibrio cholerae, enterotoxigenic Escherichia coli, and Clostridium difficile, mount neutralizing antibody responses to the toxins produced by these organisms $(12,13)$. Therefore, to study further the in vivo relevance of $H$. pylori vacuolating cytotoxin activity, we sought to detect cytotoxin-neutralizing antibodies in sera from H. pylori-infected persons.

\section{Methods}

Bacterial strains and source of vacuolating cytotoxin. Concentrated $H$. pylori culture supernatants were prepared by culturing the organism for $48 \mathrm{~h}$ in Brucella broth containing 5\% fetal bovine serum, centrifuging the culture, and concentrating the cell-free supernatant by ultrafiltration, as described previously (6). Concentrated supernatants from H. pylori strains $60190,87-199$, and 87-29 each induced vacuolation of HeLa cells in vitro (3). Vacuolating cytotoxin was purified from $H$. pylori 60190 by sequential column chromatography, as described previously (7)

Preparation of antisera. White New Zealand rabbits were immunized with concentrated supernatant from $H$. pylori 60190 (tox ${ }^{+}$) or supernatant from $H$. pylori $\mathrm{Tx} 30 \mathrm{a}\left(\right.$ tox $\left.^{-}\right)$(3). The reactivity of these sera with $H$. pylori antigens has been described previously (3).

Source of human sera. We studied $H$. pylori-infected and uninfected persons from two population groups. The first group consisted of 16 asymptomatic laboratory workers. Based on the results of a standardized enzyme-linked immunosorbent assay (ELISA) that assayed serum IgG reactivity with a pool of sonicated $H$. pylori strains (14), eight of these persons were infected with $H$. pylori and eight were not. The mean ages of the eight infected and eight uninfected persons were $38.0 \pm 1.3$ and $34.5 \pm 3.6 \mathrm{yr}$, respectively. Of the eight workers who were $H$. pylori infected, six were born outside the United States, whereas all of the uninfected workers were born within the United States. This observation is consistent with data from another study, in which foreign place of birth was associated with an increased prevalence of $H$. pylori infection (15).

The second group consisted of symptomatic patients who had previously undergone gastroduodenal endoscopy at the University Hospital and the Veterans Affairs Medical Center, Syracuse, NY. These patients were classified as $H$. pylori-infected or uninfected based on Giemsa staining and urease testing of gastric biopsy samples (CLO test, Delta 
West Limited, Weston, Australia), and evaluation of sera by ELISA (14). For this analysis, we selected $29 \mathrm{H}$. pylori-infected persons who had positive stain or CLO results and who were seropositive by ELISA, and 24 persons who were uninfected based on the results of the three assays. 8 of the $29 \mathrm{H}$. pylori-infected patients had duodenal ulcers, one had a gastric ulcer, and the remainder had nonulcer dyspepsia. Duodenal ulceration was present in one of the uninfected patients. The mean ages of the infected and uninfected patients were $66.4 \pm 2.9$ and $60.3 \pm 3.6 \mathrm{yr}$, respectively.

Neutralization of $H$. pylori cytotoxin activity. Sera were heated at $56^{\circ} \mathrm{C}$ for $30 \mathrm{~min}$, centrifuged to remove debris, and then diluted with Eagle's modified minimal essential medium (Flow Laboratories, Irvine, KA) containing 10\% fetal bovine serum (MEM-FBS). Diluted sera were incubated for $1 \mathrm{~h}$ at $37^{\circ} \mathrm{C}$ with an equal volume of concentrated $H$. pylori culture supernatant, which had been diluted 1:4 in MEM-FBS. Adherent HeLa cells $\left(10^{4}\right.$ cells per well) were then incubated for $18 \mathrm{~h}$ at $37^{\circ} \mathrm{C}$ in 96 -well plates with $50-\mu 1$ mixtures of serum and $H$. pylori supernatant plus $50 \mu \mathrm{l}$ of MEM-FBS. $H$. pylori supernatant alone or MEM-FBS alone were tested as controls. After staining for 4 min with $0.05 \%$ neutral red and washing, intravacuolar neutral red was quantitated spectrophotometrically, and was used as a measurement of cell vacuolation (6). The net optical density induced by the $H$. pylori supernatant control varied from 0.3 to 0.6 in the various experiments. Neutralization assays using purified cytotoxin were performed in the same manner, except that MEM-FBS was supplemented with 10 $\mathrm{mM}$ ammonium choride, an agent known to potentiate vacuolating cytotoxin activity (6). All assays were performed in triplicate, and results are reported as the mean optical density (OD) \pm SEM.

Purification of serum $\operatorname{IgG}$. IgG was purified from human sera using a MAbTrap G column (Pharmacia, Inc., Piscataway, NJ), dialyzed in $20 \mathrm{mM}$ sodium phosphate buffer ( $\mathrm{pH} \mathrm{7.0)}$ ), and concentrated fivefold with a 30-kD cutoff Centricon (Amicon Corp., Beverly, MA). To determine the efficiency of IgG removal from sera by this method, we employed a standardized ELISA that measured IgG reactivity with a pool of sonicated $H$. pylori strains (14). The protein concentrations of sera before and after IgG removal were determined using the Bicinchoninic Acid Protein Assay kit (Pierce Chemical Co., Rockford, IL), and were standardized at $20 \mathrm{mg} / \mathrm{ml}$. Before $\mathrm{IgG}$ removal, the mean optical density produced by $1: 200$ dilutions of five sera from $H$. pyloriinfected persons was $0.866 \pm 0.160$. After IgG removal, the mean optical density was $0.088 \pm 0.035(P=0.001)$. These data indicated that $H$. pylori-specific IgG was effectively removed by the MAbTrap G column.

Western blotting. SDS-PAGE was performed as previously described (3), using a $4.5 \%$ stacking gel and a $10 \%$ separating gel. After separation by SDS-PAGE, proteins were transferred to nitrocellulose paper by electroblotting for $1 \mathrm{~h}$ at $1 \mathrm{~A}$. Nitrocellulose paper strips were incubated for $1 \mathrm{~h}$ with 1:500 dilutions of human or rabbit sera in Tris/ saline blotting buffer (TSBB: $10 \mathrm{mM}$ Tris base, $\mathrm{pH} 8.0,0.5 \mathrm{M} \mathrm{NaCl}$, $0.5 \%$ Tween $20,0.02 \% \mathrm{NaN}_{3}$ ), washed in TSBB, and then reacted with alkaline phosphatase-conjugated anti-human IgG (Tago, Inc., Burlingame, CA) or anti-rabbit IgG (Boehringer Mannheim Biochemicals, Indianapolis, IN). Proteins were resolved using an immunoenzymatic method (16).

ELISA. Human sera were tested for reactivity with the purified cytotoxin in an ELISA, as described previously (7). Sera were diluted 1:100 and reacted with $15 \mathrm{ng}$ purified cytotoxin per microtiter well. Peroxidase-conjugated anti-human IgG (Tago, Inc.) was used as the conjugate.

Statistical methods. Distributions of optical density values were compared using a two-tailed Student's $t$ test for independent variables.

\section{Results}

Neutralization of $H$. pylori cytotoxin activity by immune rabbit serum. We first sought to determine whether immunization with $H$. pylori cytotoxin induced production of neutralizing antibodies. In high concentrations, all the sera tested partially neutralized cytotoxin activity (Fig. 1). However, serum from a rabbit immunized with tox ${ }^{+}$supernatant neutralized $H$. pylori cytotoxin activity significantly better than preimmune serum $(P<0.05$ at dilutions of $1: 2$ to $1: 128)$. Immunization with tox ${ }^{-}$supernatant did not produce a neutralizing antibody response. These results indicate that immunization with culture supernatant from a tox ${ }^{+} H$. pylori strain induces a neutralizing antibody response to the cytotoxin.

Neutralization of $H$. pylori cytotoxin activity by human sera. To determine whether neutralizing antibodies to the cytotoxin are present in sera from $H$. pylori-infected humans, sera from 16 asymptomatic laboratory workers were studied (Fig. 2 ). At dilutions of $1: 2$ to $1: 16$, sera from three of the eight infected persons ( $\mathrm{A} 1, \mathrm{~K} 1$, and $\mathrm{W} 1$ ) completely neutralized cytotoxin activity, an effect not produced by any of the sera from uninfected persons. These results indicated the range of serum dilutions that were appropriate for use in subsequent experiments.

Antigenic relatedness of vacuolating cytotoxins from $H$. pylori strains. We next sought to determine whether the cytotoxins produced by different $H$. pylori strains were antigenically related. Four sera from $H$. pylori-infected persons (including $\mathrm{A} 1, \mathrm{~K} 1$, and $\mathrm{W} 1$ ) and four sera from uninfected persons were each reacted with culture supernatants from distinct tox ${ }^{+} H$. pylori strains (Fig. 3). The sera from three of the four $H$. pylori-infected persons completely neutralized the cytotoxins produced by each of the tox ${ }^{+} H$. pylori strains tested, whereas the sera from the four uninfected persons failed to neutralize cytotoxin activity or produced only partial neutralization. Similarly, rabbit antiserum to tox ${ }^{+}$supernatant from $H$. pylori 60190 neutralized the toxins produced by each of the tox ${ }^{+} H$. pylori strains, whereas preimmune serum did not (data not shown). In contrast, none of the sera neutralized the vacuola-

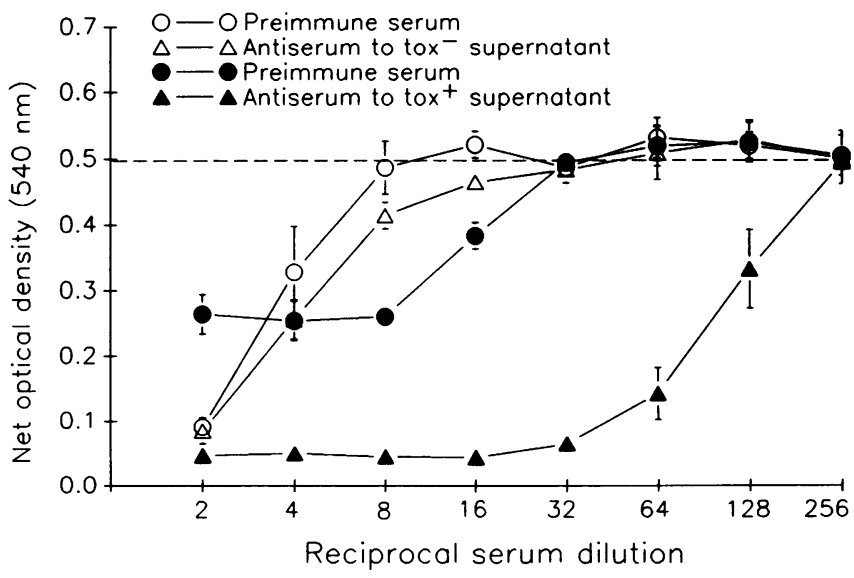

Figure 1. Neutralization of $H$. pylori cytotoxin activity by rabbit antiserum. White New Zealand rabbits were immunized with culture supernatant from $H$. pylori 60190 (tox $^{+}$) or $\mathrm{Tx} 30 \mathrm{a}$ (tox ${ }^{-}$). The preimmune and immune sera were then tested for cytotoxin-neutralizing activity. The net optical density produced by supernatant from H. pylori 60190 in this experiment is indicated by the dashed line (- - - -). At dilutions of $1: 2$ to $1: 128$, serum from the rabbit immunized with tox ${ }^{+} H$. pylori supernatant neutralized cytotoxin activity significantly better than preimmune serum $(P<0.05)$. The neutralizing activities did not differ in preimmune and immune sera from the rabbit immunized with tox ${ }^{-}$supernatant. 


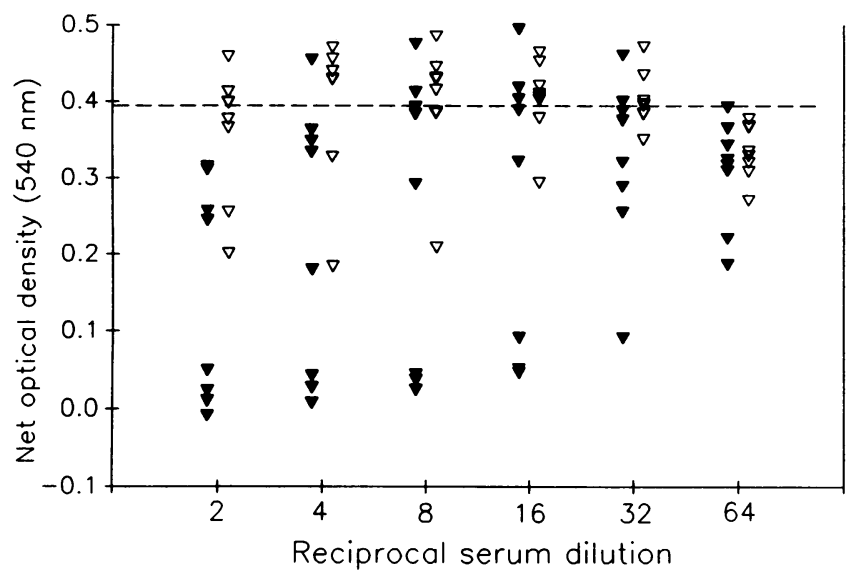

Figure 2. Neutralization of $H$. pylori cytotoxin activity by sera from asymptomatic humans. Dilutions of sera from eight asymptomatic $H$. pylori-infected persons $(\nabla)$ and eight uninfected $(\nabla)$ persons were tested for cytotoxin-neutralizing activity. The net optical density produced by supernatant from $H$. pylori 60190 in this experiment is indicated by the dashed line. When diluted 1:2-1:16, sera from three of the infected persons completely neutralized $H$. pylori cytotoxin activity.

tion produced by trimethylamine, a weak base known to induce intracytoplasmic vacuole formation (17). These data indicate that the vacuolating cytotoxins produced by different $H$. pylori strains are antigenically related, and that the human serum antibody response is directed toward conserved epitopes. The failure of human or rabbit immune sera to neutralize trimethylamine activity indicates that the neutralization of cytotoxin activity by these sera is a specific effect, and suggests

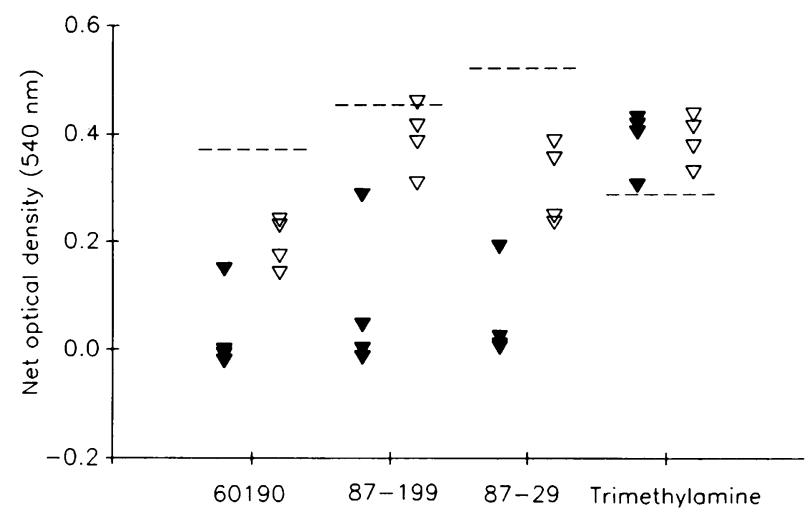

Figure 3. Antigenic relatedness of cytotoxins produced by various $H$. pylori strains. Sera from $H$. pylori-infected $(\nabla)$ and uninfected $(\nabla)$ persons were tested for the capacity to neutralize cell vacuolation induced by $H$. pylori culture supernatants or trimethylamine. Supernatants were prepared from three $H$. pylori strains that produced vacuolating cytotoxins $(60190,87-199$, and $87-29)$. The net optical densities produced by the supernatants alone or trimethylamine alone are indicated by the dashed lines. Sera from three of the four $H$. pylori-infected persons ( $\mathrm{A} 1, \mathrm{~K} 1$, and $\mathrm{W} 1$ ) completely neutralized the activity of cytotoxins produced by each of these $H$. pylori strains, whereas sera from the four uninfected persons did not. All of the sera failed to neutralize the vacuolation induced by trimethylamine, an indication that the neutralizing activity in human serum was directed specifically directed toward $H$. pylori cytotoxin. that neutralizing antibodies are directed specifically toward $H$. pylori cytotoxin.

IgG-mediated neutralization of cytotoxin activity. To determine whether the cytotoxin-neutralizing activity in human sera was antibody mediated, IgG was purified from the sera of five asymptomatic infected and five uninfected persons, using a MAb Trap G column. The cytotoxin-neutralizing activity of whole sera from these persons is shown in Fig. 4 (top). At a protein concentration of $12.5 \mathrm{mg} / \mathrm{ml}$, the sera from the infected persons showed significantly greater neutralizing activity than sera from uninfected persons $(P=0.03)$. Purified IgG from the sera of each of the five infected persons neutralized $H$. pylori cytotoxin activity, whereas IgG from the 5 uninfected persons did not (Fig. 4, middle); this difference was significant $(P<0.05)$ at protein concentrations $\geq 0.76 \mathrm{mg} / \mathrm{ml}$. These results indicate that specific cytotoxin-neutralizing IgG antibodies are present in sera from $H$. pylori-infected persons. After removal of IgG, the remaining components of sera from $H$. pylori-infected persons as well as from uninfected persons partially neutralized cytotoxin activity to a similar degree (Fig. 4 , bottom). The latter activity presumably contributes to the low-level cytotoxin-neutralizing activity present in sera from persons not infected with $H$. pylori.

Association between neutralization of cytotoxin activity and serologic recognition of an $87-k D H$. pylori protein. We hypothesized that neutralization of cytotoxin activity by sera might be associated with immunoblot recognition of a specific $H$. pylori protein. To test this hypothesis, supernatant from $H$. pylori 60190 was immunoblotted with sera from the eight asymptomatic $H$. pylori-infected persons described previously, as well as with the immune rabbit serum that neutralized cytotoxin activity (Fig. 5). The antigen used in these Western blotting studies was prepared by culturing $H$. pylori 60190 in Brucella broth containing $0.5 \%$ charcoal for $48 \mathrm{~h}$; after centrifugation, the supernatant was concentrated 30 -fold by precipitation with a $50 \%$ saturated solution of ammonium sulfate. The three human sera with the strongest neutralizing activity (A1, W1, and $\mathrm{K} 1$ ) all strongly recognized an $87-\mathrm{kD}$ band, which was also recognized by rabbit antisera to tox ${ }^{+} H$. pylori supernatant. Thus, high-titer cytotoxin-neutralizing activity in human or rabbit sera was associated with the presence of $\mathrm{IgG}$ antibodies to an $87-\mathrm{kD}$ protein.

Neutralization of the purified $H$. pylori cytotoxin by human sera. The vacuolating cytotoxin has recently been purified to homogeneity from $H$. pylori culture supernatant, and migrates as an $87-\mathrm{kD}$ band under denaturing conditions (7). To study further the neutralization of vacuolating cytotoxin activity by human sera, we tested sera from 53 well-characterized patients for the capacity to neutralize the activity of the purified cytotoxin. Owing to limited quantities of sera available, cytotoxin neutralization was assessed using only a 1:8 dilution of sera. The net optical density produced by the purified cytotoxin alone was $0.276 \pm 0.04$. The mean net optical densities produced by purified cytotoxin after preincubation with sera from 29 infected or 24 uninfected persons were $0.002 \pm 0.01$ and $0.045 \pm 0.01$, respectively ( $P=0.007$, Student's $t$ test ). Thus, sera from both groups of patients neutralized cytotoxin activity, but sera from $H$. pylori-infected persons as a group neutralized to a significantly greater extent than sera from uninfected persons. The cytotoxin neutralizing activity of seven (24\%) of the sera from the 29 infected persons was significantly $(>2$ 

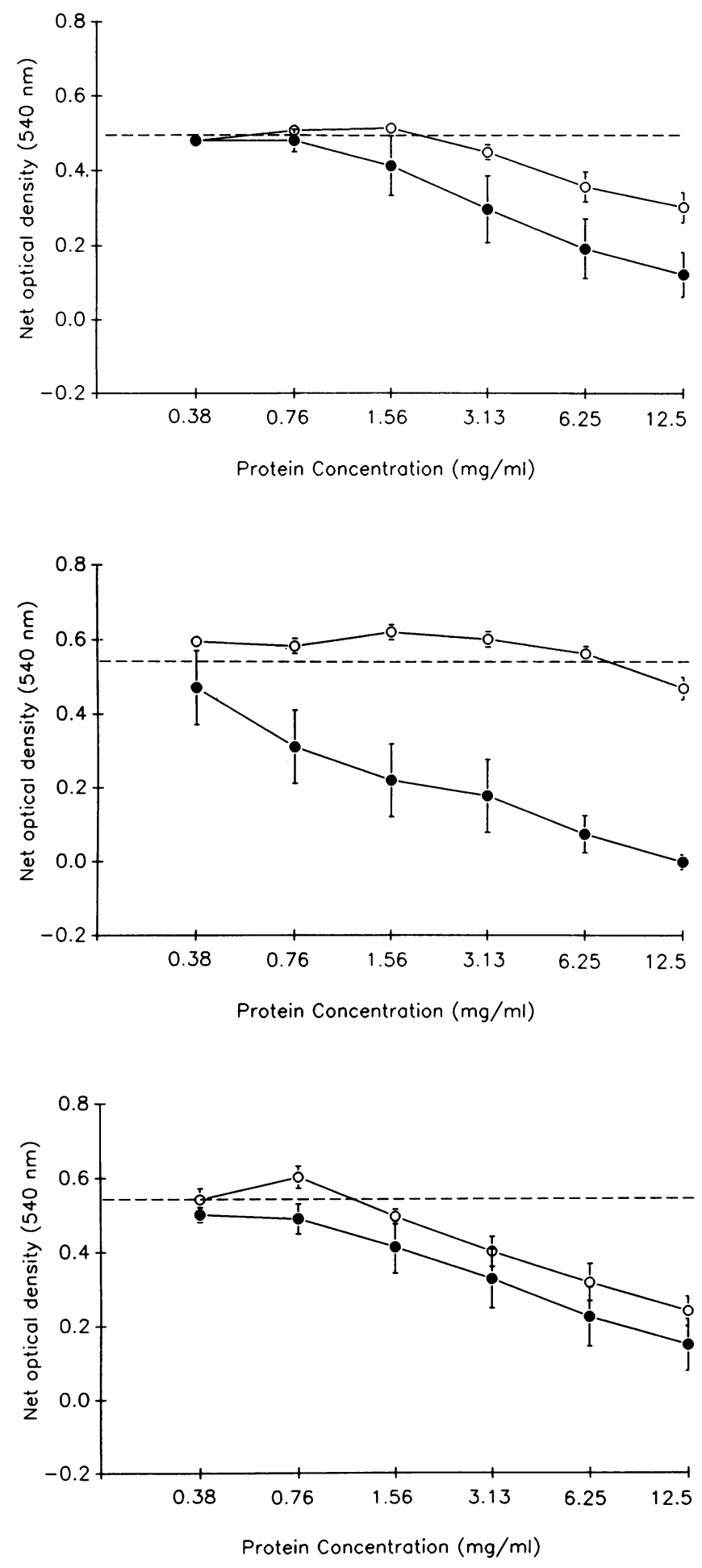

Figure 4. Neutralization of $H$. pylori cytotoxin activity by whole human sera, serum IgG, and sera after removal of IgG. IgG was purified from the sera of five asymptomatic $H$. pylori-infected persons $(\bullet)$ and five uninfected persons ( $O$ ) using a MAbTrap G column. The whole sera (top), purified IgG (middle), and sera after removal of IgG (bottom) were then tested for cytotoxin-neutralizing activity. The net optical density produced by supernatant from $H$. pylori 60190 alone is indicated by the dashed line. The purified serum IgG from the five $H$. pylori-infected persons completely neutralized cytotoxin activity, whereas IgG from uninfected persons did not. After removal of IgG, sera from infected and uninfected persons partially neutralized cytotoxin activity to similar degrees.

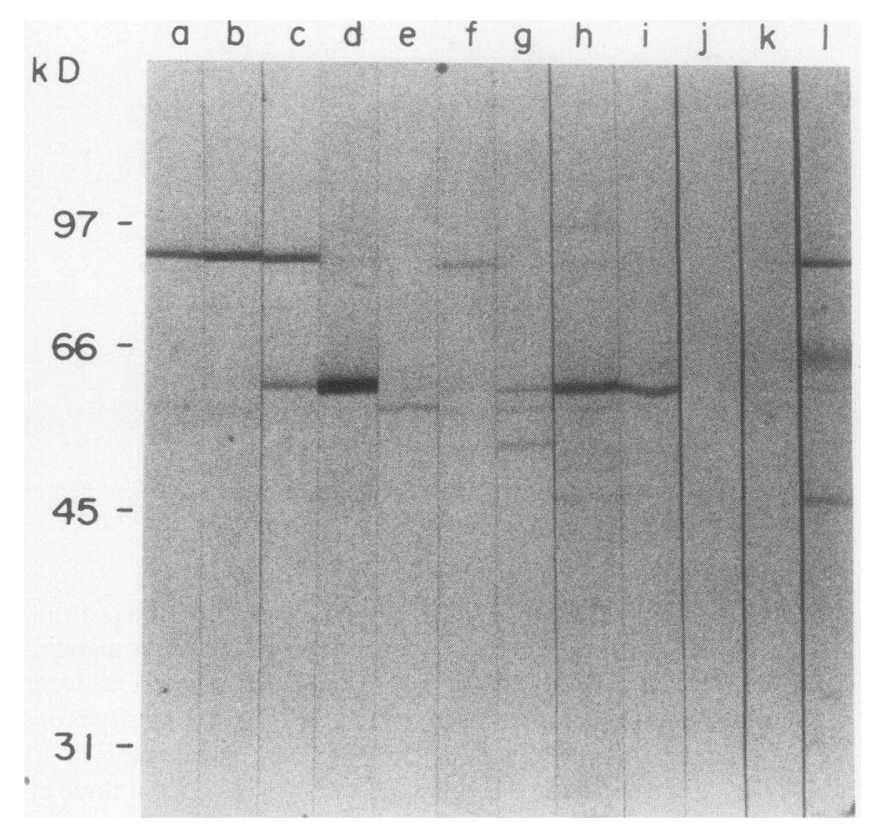

Figure 5. Immunoblot of concentrated supernatant from $H$. pylori 60190. Concentrated supernatant from $H$. pylori 60190 was immunoblotted with sera from eight $H$. pylori-infected persons (lanes $a-h$ ), two uninfected persons (lanes $i$ and $j$ ), preimmune rabbit serum (lane $k$ ), and antiserum to tox ${ }^{+}$supernatant from $H$. pylori 60190 (lane l). Diluted 1:500, sera from three $H$. pylori-infected persons (A1, K1, W1) that strongly neutralized cytotoxin activity (Fig. 2) strongly recognized an $87-\mathrm{kD}$ band (lanes $a-c$ ). The $87-\mathrm{kD}$ band also was recognized by a 1:500 dilution of rabbit antiserum to tox ${ }^{+}$supernatant (lane $l$ ), but not preimmune serum (lane $k$ ). The $87-\mathrm{kD}$ band was weakly recognized by the serum in lane $f$. Thus, high-titer cytotoxin-neutralizing activity was associated with strong recognition of an $87-\mathrm{kD}$ protein in immunoblotting studies.

SD) greater than the mean neutralizing activity of sera from uninfected persons, whereas none of the sera from the 24 uninfected persons produced this degree of neutralization ( $P$ $=0.01$, Fisher's exact test ).

Association between cytotoxin neutralization and ELISA reactivity with the purified cytotoxin. To determine the prevalence of IgG antibodies to the cytotoxin, sera from the 53 patients described above were tested in an ELISA for reactivity with the purified cytotoxin, prepared by sequential column chromatography as described previously (7) (Fig. 6, top). The mean reactivity of sera from $H$. pylori-infected persons with the purified cytotoxin was significantly greater than that of sera from uninfected persons $(P<0.0001)$. Recognition of the purified toxin by sera from $18(62 \%)$ of the 29 infected persons was significantly ( $>2 \mathrm{SD}$ ) greater than the mean optical density value produced by sera from the 24 uninfected persons. To determine the relationship between neutralization of the purified toxin in the previous experiment and $\mathrm{IgG}$ reactivity with the purified toxin in the ELISA, a linear regression analysis was performed (Fig. 6, bottom). For sera from $H$. pylori-infected persons, a significant relationship was present $(P<0.0001, r$ $=0.734$ ). Interestingly, for sera from uninfected persons, a similar but less significant trend was observed $(P=0.003, r$ $=0.583$ ). These data indicate a strong relationship between neutralization of the purified cytotoxin and IgG reactivity with the purified cytotoxin. 

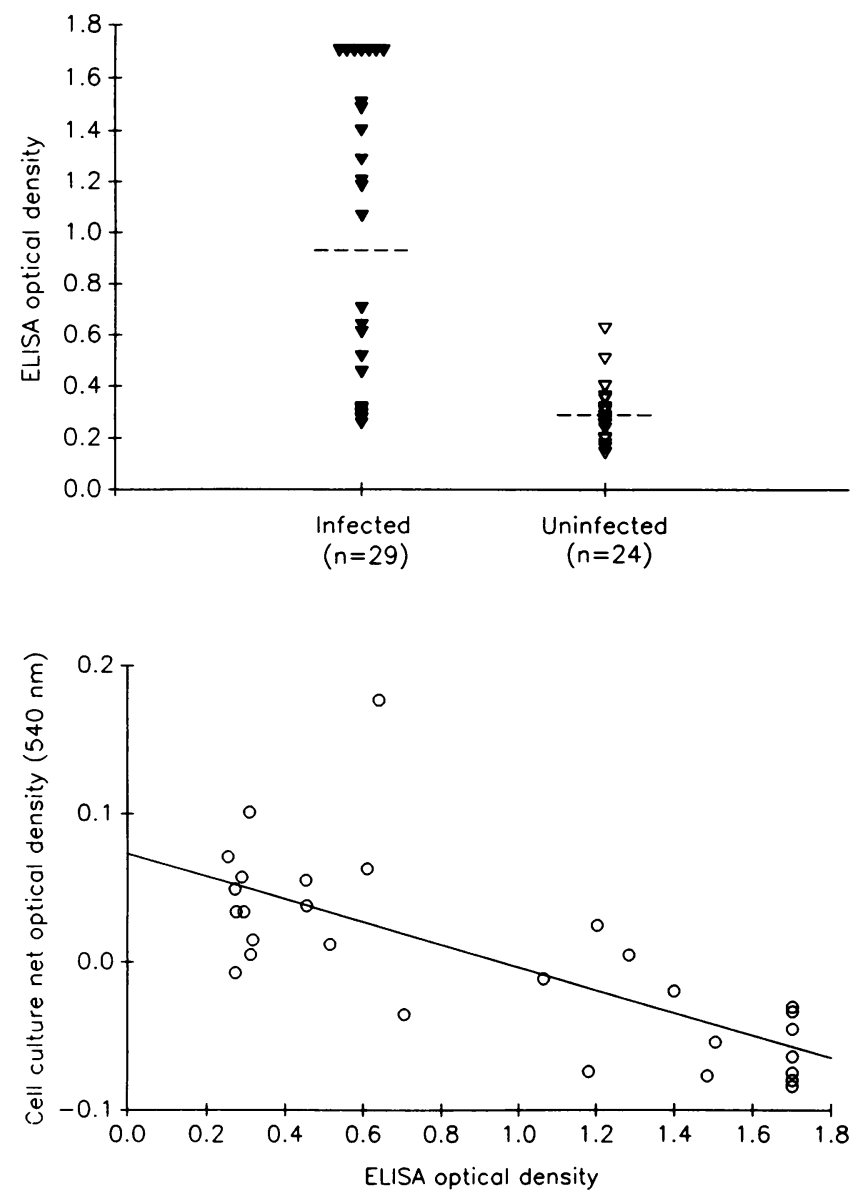

Figure 6 . Association between cytotoxin neutralizing activity and serologic recognition of the purified cytotoxin. Sera from $29 \mathrm{H}$. pylori-infected persons and 24 uninfected persons were tested in an ELISA for IgG reactivity with the purified cytotoxin. (Top) As a group, the reactivity of sera from $H$. pylori-infected persons was significantly greater than that of sera from uninfected persons (mean optical densities $0.930 \pm 0.110$ vs. $0.287 \pm 0.023, P<0.0001$ ). (Bottom) The IgG reactivity of sera from the $29 \mathrm{H}$. pylori-infected persons with the purified cytotoxin in an ELISA was significantly associated with neutralization of cytotoxin activity in the cell culture assay ( $P$ $<0.0001, r=0.734$ by linear regression analysis).

\section{Discussion}

Although the majority of persons infected with $H$. pylori are asymptomatic, infection is a risk factor for the development of peptic ulceration $(1,2)$ and possibly gastric carcinoma $(18-$ 20 ). One of the determinants that may contribute to the virulence of $H$. pylori infection is a cytotoxin that induces vacuolation of eukaryotic cells $(3,4)$. The cytotoxin has recently been purified, and corresponds to an $87-\mathrm{kD}$ band visualized by SDSPAGE under denaturing conditions (7). Based on detection in a cell culture assay, approximately $50-60 \%$ of $H$. pylori strains produce the cytotoxin in vitro (3-5). Two studies have demonstrated that patients with duodenal ulceration are more commonly infected with cytotoxin-producing $H$. pylori strains than infected patients with gastritis only $(21,22)$. To determine whether the vacuolating cytotoxin is produced by $H$. pylori in vivo, we sought to detect neutralizing antibodies to the cytotoxin in sera from $H$. pylori-infected persons.
In this study, cytotoxin neutralizing activity was detectable in sera from $H$. pylori-infected humans in serum dilutions ranging from $1: 2$ to $1: 16$, and significantly greater cytotoxinneutralizing activity was present in sera from $H$. pylori-infected persons than in sera from uninfected persons. These data are consistent with the results of Leunk et al. (23), who reported neutralization of $H$. pylori cytotoxin activity by human sera diluted in a range from 1:2 to 1:64. In this study, we have demonstrated that cytotoxin activity is neutralized by the purified IgG fraction of sera from $H$. pylori-infected but not uninfected persons. Vacuolating cytotoxin activity was also partially neutralized by human sera after removal of IgG. The presence of the latter activity in sera from both $H$. pylori-infected and uninfected persons suggests that this effect is not mediated by $H$. pylori-specific antibodies. This phenomenon may be mediated by a variety of serum components, including amino acids and hormones, which are known to inhibit autophagic vacuole formation and cellular protein degradation (24-26).

The current study has demonstrated that neutralization of vacuolating cytotoxin activity by human sera is associated with the presence of IgG antibodies to the purified $H$. pylori cytotoxin. We speculate that the presence of neutralizing antibodies to the cytotoxin reflects current or recent infection with a tox ${ }^{+}$ H. pylori strain. Based upon detection in the cell culture assay, $24 \%$ of sera from $\mathrm{H}$. pylori-infected persons contained cytotoxin neutralizing antibodies. In contrast, $\mathrm{IgG}$ reactivity with the purified cytotoxin in an ELISA was demonstrated for $62 \%$ of the sera from $\mathrm{H}$. pylori-infected persons. These data suggest that the ELISA is a more sensitive method for detecting anticytotoxin antibodies than the neutralization assay. The recognition of cytotoxin in the ELISA by sera from $62 \%$ of $\mathrm{H}$. pyloriinfected persons may be interpreted as evidence for cytotoxin production in vivo by this proportion of strains; a similar proportion of strains produce detectable cytotoxin activity in vitro $(3,4)$.

It is notable that a weaker relationship between cytotoxin neutralization and ELISA reactivity with the purified cytotoxin was observed among sera from uninfected persons. This phenomenon suggests that the vacuolating cytotoxin may have a weak antigenic relationship with an antigen to which all persons are commonly exposed. There is partial homology between the amino-terminal sequence of the $H$. pylori cytotoxin and several bacterial transport or ion channel proteins (7); such proteins are potential candidates for this antigenic similarity.

In conclusion, the presence of cytotoxin-neutralizing antibodies in sera from persons infected with $H$. pylori provides strong evidence that the cytotoxin is produced in vivo. It is not yet known whether the cytotoxin of $H$. pylori plays an important role in the pathogenesis of infection, or whether neutralizing antibodies to the cytotoxin modulate the course of infection. In the future, these questions may be studied in animal models, using isogenic $H$. pylori strains differing only in cytotoxin activity, or by studies involving immunization with the cytotoxin.

\section{Acknowledgments}

Timothy L. Cover is an Associate Investigator of the Department of Veterans Affairs and was a recipient of a Searle Scholars Fellowship 
Award from the Infectious Disease Society of America. This work was supported in part by a grant from The Procter \& Gamble Company.

\section{References}

1. Cover, T. L., and M. J. Blaser. 1992. Helicobacter pylori and gastroduodenal disease. Annu. Rev. Med. 43:135-146.

2. Petersen, W. L. 1991. Helicobacter pylori and peptic ulcer disease. N. Engl. J. Med. 324:1043-1048.

3. Cover, T. L., C. P. Dooley, and M. J. Blaser. 1990. Characterization of and human serologic response to proteins in Helicobacter pylori broth culture supernatants with vacuolizing cytotoxin activity. Infect. Immun. 58:603-610.

4. Leunk, R. D., P. T. Johnson, B. C. David, W. G. Kraft, and D. R. Morgan. 1988. Cytotoxic activity in broth culture filtrates of Campylobacter pylori. $J$. Med. Microbiol. 26:93-99.

5. Figura, N., P. Guglielmetti, A. Rossolini, A. Barberi, G. Cusi, R. A. Musmanno, M. Russi, and S. Quaranta. 1989. Cytotoxin production by Campylobacter pylori strains isolated from patients with peptic ulcers and from patients with chronic gastritis only. J. Clin. Microbiol. 27:225-226.

6. Cover, T. L., W. Puryear, G. I. Perez-Perez, and M. J. Blaser. 1991. Effect of urease on HeLa cell vacuolation induced by Helicobacter pylori cytotoxin. Infect. Immun. 59:1264-1270.

7. Cover, T. L., and M. J. Blaser. 1992. Purification and characterization of the vacuolating toxin from Helicobacter pylori. J. Biol. Chem. 267:10570-10575.

8. Eaton, K. A., D. R. Morgan, and S. Krakowka. 1989. Campylobacter pylori virulence factors in gnotobiotic piglets. Infect. Immun. 57:1119-1125.

9. Tricottet, V., P. Bruneval, O. Vire, and J. P. Camilleri. 1986. Campylobacter-like organisms and surface epithelium abnormalities in active, chronic gastritis in humans: an ultrastructural study. Ultrastruct. Pathol. 10:113-122.

10. Hessey, S. J., J. Spencer, J. I. Wyatt, G. Sobala, B. J. Rathbone, A. T. R. Axon, and M. F. Dixon. 1990. Bacterial adhesion and disease activity in Helicobacter associated chronic gastritis. Gut. 31:134-138.

11. Thomsen, L. L., J. B. Gavin, and C. Tasman-Jones. 1990. Relation of Helicobacter pylori to the human gastric mucosa in chronic gastritis of the antrum. Gut. 31:1230-1236.

12. Nakamura, S., M. Mikawa, S. Nakashio, M. Takabatake, I. Okado, K. Yamakawa, T. Serikawa, S. Okumura, and S. Nishida. 1981. Isolation of Clostridium difficile from the feces and the antibody in sera of young and elderly adults. Microbiol. Immunol. 25:345-351.
13. Sack, R. B., B. Jacobs, and R. Mitra. 1974. Antitoxin responses to infections with enterotoxigenic Escherichia coli. J. Infect. Dis. 129:330-335.

14. Perez-Perez, G. I., B. M. Dworkin, J. E. Chodos, and M. J. Blaser. 1988. Campylobacter pylori antibodies in humans. Ann. Intern. Med. 109:11-17.

15. Perez-Perez, G. I., S. S. Witkin, M. D. Decker, and M. J. Blaser. 1991. Seroprevalence of Helicobacter pylori infection in couples. J. Clin. Microbiol. 29:642-644.

16. Blake, M. S., K. H. Johnston, G. I. Russel-Jones, and E. C. Gotschlich. 1984. A rapid, sensitive method for detection of alkaline phosphatase-conjugated anti-antibody on western blots. Anal. Biochem. 136:175-179.

17. Okhuma, S., and B. Poole. 1981. Cytoplasmic vacuolation of mouse peritoneal macrophages and the uptake into lysosomes of weakly basic substances. $J$. Cell Biol. 90:656-664.

18. Parsonnet, J., G. D. Friedman, D. P. Vandersteen, Y. Chang, J. H. Vogelman, N. Orentreich, and R. K. Sibley. 1991. Helicobacter pylori infection and the risk of gastric carcinoma. 1991. N. Engl. J. Med. 325:1127-1131.

19. Nomura, A., G. N. Stemmermann, P.-H. Chyou, I. Kato, G. I. PerezPerez, and M. J. Blaser. 1991. Helicobacter pylori infection and gastric carcinoma among Japanese-Americans in Hawaii. $N$. Engl. J. Med. 325:1132-1136.

20. Talley, N. J., A. R. Zinsmeister, E. P. DiMagno, A. Weaver, H. A. Carpenter, G. I. Perez-Perez, and M. J. Blaser. 1991. Gastric adenocarcinoma and Helicobacter pylori infection. J. Natl. Cancer Inst. 83:1734-1739.

21. Figura, N. P. Guglielmetti, A. Rossolini, A. Barberi, G. Cusi, R. A. Musmanno, M. Russi, and S. Quaranta. 1989. Cytotoxin production by Campylobacter pylori strains isolated from patients with peptic ulcers and from patients with chronic gastritis only. J. Clin. Microbiol. 27:225-226.

22. Goosens, H., L. Vlaes, J.-P. Lambert, Y. Glupczynski, A. Burette, and J.-P. Butzler. 1991. In vitro cytotoxin production by Helicobacter pylori strains and clinical correlations. Microb. Ecol. Health Dis. 4:S130.

23. Leunk, R. D., M. A. Ferguson, D. R. Morgan, D. E. Low, and A. E. Simor. 1990. Antibody to cytotoxin in infection by Helicobacter pylori. J. Clin. Microbiol. 28:1181-1184

24. Pfeifer, U., E. Werder, and H. Bergeest. 1978. Inhibition by insulin of the formation of autophagic vacuoles in rat liver: a morphometric approach to the kinetics of intracellular degradation by autophagy. J. Cell Biol. 78:152-167.

25. Ballard, F. J., S. E. Knowles, S. S. C. Wong, J. B. Bodner, C. M. Wood, and J. M. Gunn. 1980. Inhibition of protein breakdown in culture cells is a consistent response to growth factors. FEBS (Fed. Eur. Biochem Soc.) Lett. 114:209-212.

26. Schworer, C. M., K. A. Shiffer, and G. E. Mortimore. 1981. Quantitative relationship between autophagy and proteolysis during graded amino acid deprivation in perfused rat liver. J. Biol. Chem. 256:7652-7658. 